\title{
An improved trigger for Askaryan radio detectors
}

\author{
Christian Glaser $^{a, b, *}$ and Steven W. Barwick ${ }^{b}$ \\ ${ }^{a}$ Uppsala University Department of Physics and Astronomy, Uppsala, SE-752 37, Sweden \\ ${ }^{b}$ Department of Physics and Astronomy, University of California, Irvine, CA 92697, USA \\ E-mail: christian.glaser@physics.uu.se, sbarwick@uci.edu
}

High-energy neutrinos with energies above a few $10^{16} \mathrm{eV}$ can be measured efficiently with in-ice radio detectors which complement optical detectors such as IceCube at higher energies. Several pilot arrays explore the radio technology successfully in Antarctica. Because of the low flux and interaction cross-section of neutrinos it is vital to increase the sensitivity of the radio detector as much as possible. In this manuscript, different approaches to trigger on high-energy neutrinos are systematically studied and optimized. We find that the sensitivity can be improved substantially (by more than $50 \%$ between $10^{17} \mathrm{eV}$ and $10^{18} \mathrm{eV}$ ) by simply restricting the bandwidth in the trigger to frequencies between $80 \mathrm{MHz}$ and $200 \mathrm{MHz}$ instead of the currently used $80 \mathrm{MHz}$ to $\sim 1 \mathrm{GHz}$ bandwidth. These findings inform the detector design of future Askaryan detectors and can be used to increase the sensitivity to high-energy neutrinos significantly without any additional costs. The findings also apply to the phased array trigger concept.

$37^{\text {th }}$ International Cosmic Ray Conference (ICRC 2021)

July 12 th - 23rd, 2021

Online - Berlin, Germany

\footnotetext{
*Presenter
} 


\section{Introduction}

The low flux of ultra-high energy (UHE) neutrinos paired with the small interaction cross section of neutrinos makes is challenging to construct a sensitive enough detector able to measure UHE neutrinos at a reasonable rate. With the radio technique, huge volumes of ice can be instrumented at moderate costs through a sparse array of radio detector stations owing to the large attenuation length of $O(1 \mathrm{~km})$ radio waves in ice.

The feasibility of the radio technique is demonstrated by the two pilot arrays ARIANNA (see e.g. [1]) installed on the Ross Ice Shelf and at the South Pole, and ARA at the South Pole [2]. Larger in-ice radio arrays are currently in the planning and/or construction phase. The RNO-G project will install 35 autonomous radio detector stations in central Greenland [3]. The ARIANNA-200 project plans to install 200 autonomous radio detector stations on the Ross Ice Shelf, Antarctica which would uniquely survey the vast majority of the southern sky at any instant in time due to the reflective properties of the ice-water interface at the bottom of the ice shelf [4]. An order of magnitude larger radio detector is part of the suggested IceCube-Gen2 detector to increase the energy reach towards higher energies [5].

Still, the expected event rates are small and physics results will likely be dominated by statistics. Therefore, the sensitivity of each radio detector station is increased as much as possible which is challenging as current pilot arrays already have such low trigger thresholds that the vast majority of triggers are thermal noise fluctuations. One way to increase the sensitivity is to deploy multiple dipole antennas in close proximity and combine them in an interferometric phased array to increase the effective signal-to-noise ratio [6]. Another approach is to reject thermal noise fluctuations in real time which also allows to further reduce the trigger thresholds [7]. Here, we present a simpler but equally effective way to increase the sensitivity that can be implemented with negligible additional costs: Restricting the bandwidth in the trigger channels to the lower part of the typical bandwidth, i.e., $80-200 \mathrm{MHz}$ instead of $80-800 \mathrm{MHz}$, increases the sensitivity by more than $50 \%$ which we will discuss in the following. This is complementary to the other techniques to increase sensitivity mentioned before, i.e., the techniques can be combined to further increase the overall sensitivity of the detector. For a more detailed presentation that also includes the discussion of different trigger schemes, please refer to [8].

\section{Method}

To study the effect of bandwidth on the sensitivity, we first need to develop a method to equally compare different trigger schemes. For all practical purposes, the trigger rate on thermal noise fluctuations sets the thresholds, where the trigger rate is determined by the capabilities of the detector and in particular the data transfer systems. If one relies on Iridium satellite communications, the acceptable event rate is $O(1 \mathrm{mHz})$.

In the following, we will study the effect of bandwidth exemplarily for the trigger logic of the ARIANNA detector but our findings also apply to other trigger schemes as discussed in [8]. The ARIANNA trigger is fulfilled if the amplitude in one readout channel is both above and below a threshold $T$ within a time window $\Delta t$. This trigger scheme exploits the property that the electric field of an Askaryan pulse is nearly bipolar, and the maximum positive and negative amplitudes 


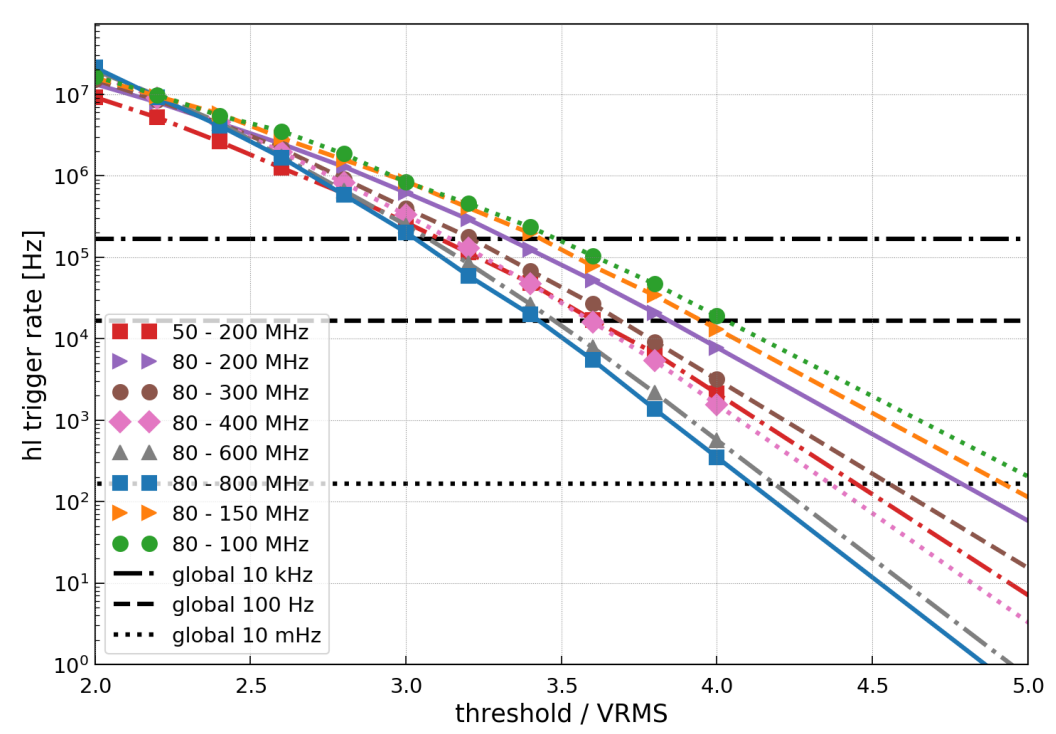

Figure 1: Single channel high/low trigger rate as a function of threshold normalized to the noise RMS for a variety of different bandwidths as indicated by the legend. The lines show a linear interpolation/extrapolation to the simulated data points. Horizontal lines show the resulting global trigger rate for a 2 out of 4 majority logic with a coincidence time of $30 \mathrm{~ns}$ between different channels.

become more symmetric after antenna and amplifier response functions are considered, whereas thermal noise fluctuations are often only one-sided. In addition, a temporal coincidence between two out of the four LPDA antennas is required which further reduces the trigger rate.

A variety of different bandwidths between $80 \mathrm{MHz}$ and $800 \mathrm{MHz}$ are studied by applying a commonly available Butterworth high pass and low pass filter. All high-pass filters are modelled using a 5th order Butterworth filter, and all low-pass filters are modelled using a 10th order Butterworth filter. The bandwidth is specified by quoting the critical frequencies, i.e., the frequency where the filter response drops to $-3 \mathrm{~dB}$. In the following, we restrict ourselves to present only the results for an upper cutoff frequency of $200 \mathrm{MHz}, 400 \mathrm{MHz}$ and $800 \mathrm{MHz}$ to focus on the main general dependencies and because we found that additional changes of the lower cutoff frequency and/or further finetuning of the cutoff frequencies has little impact.

The resulting single-channel trigger rates as a function of threshold normalized to the RMS noise are shown in Fig. 1 for a variety of different bandwidths. The corresponding single-channel trigger rates for a global trigger rate of $10 \mathrm{kHz}, 100 \mathrm{~Hz}$ and $10 \mathrm{mHz}$ are indicated with horizontal lines in Fig. 1.

For the high/low trigger, we observe the general trend that the trigger rate increases with decreasing bandwidth for the same threshold/RMS noise ratio. This is because an upward thermal noise fluctuation always has a small downward fluctuation on both sides because of the bandwidth limitation. This mexican-hat like waveform gets more pronounced as the bandwidth is reduced. This results in a higher probability to produce an opposite sign fluctuation, which increases the high/low trigger rate. We will see later that the sensitivity to neutrinos is increased with a decreased bandwidth despite the higher trigger thresholds. 


\section{Effect of trigger bandwidth on sensitivity}

In this section we answer the question: What is the impact of bandwidth on the neutrino sensitivity for a given trigger scheme and detector response. Current experiments such as ARA and ARIANNA use a large bandwidth of $80 \mathrm{MHz}$ to about $1 \mathrm{GHz}$ which is motivated by the prediction that the Askaryan signal extends up to $\mathrm{GHz}$ frequencies if observed on the Cherenkov cone. The sensitivity of the detector is quantified in terms of effective volume to an isotropic neutrino flux. It is given by the weighted sum of all triggered events divided by the total number of events multiplied by the simulation volume and the simulated solid angle. The weighting factor is the probability of a neutrino reaching the simulation volume (and not being absorbed by the Earth). The effective volume is directly proportional to the number of observed neutrinos.

\subsection{Simulation settings}

We perform this study for the proposed ARIANNA-200 detector, an array of 200 autonomous detector stations on the Ross Ice Shelf which uses two orthogonal pairs of downward pointing LPDA antennas to measure and trigger on Askaryan signals [4]. An additional bicone antenna (as used by the ARA detector) at a depth of $10 \mathrm{~m}$ is also part of the station to aid event reconstruction [9] but not used for the trigger.

The sensitivity to neutrinos for each trigger bandwidth and trigger scheme is simulated using NuRadioMC [10] that uses NuRadioReco for the detector simulation [11]. We perform the simulation with the ice properties of the Moore's Bay site on the Ross Ice Shelf. We use the Alvarez2000 Askaryan emission model and assume a noise temperature of $250 \mathrm{~K}$. We assume a 1:1:1 flavor ratio between electron, muon and tau neutrinos, distribute arrival directions isotropically, and place the neutrino interactions uniformly in the ice around the detector. The neutrino sensitivity is simulated at discrete neutrino energies and quantified via the effective volume per neutrino energy.

\subsection{Impact of bandwidth using the high/low trigger scheme}

According to the procedure outlined in Sec. 2, we first calculate the threshold that corresponds to a global thermal noise trigger rate of $100 \mathrm{~Hz}$ (cf. Fig. 1). We find a threshold of approximately 3.4 times the RMS noise for the largest studied bandwidth of $80 \mathrm{MHz}-800 \mathrm{MHz}$. The trigger threshold increases for decreasing bandwidth. For the smallest bandwidth of $80 \mathrm{MHz}-200 \mathrm{MHz}$ we obtain a trigger threshold of approximately 4 times the RMS noise.

In Fig. 2, the increase in effective volume of a detector with a trigger bandwidth of $80 \mathrm{MHz}-$ $200 \mathrm{MHz}$ and $80 \mathrm{MHz}-400 \mathrm{MHz}$ with respect to the reference with a large bandwidth of $80 \mathrm{MHz}$ $800 \mathrm{MHz}$ is shown. We find that the sensitivity of an Askaryan detector can be increased by $50 \%$ for neutrino energies between $10^{17} \mathrm{eV}$ and $10^{18} \mathrm{eV}$ with a simple bandpass filter despite running at a higher threshold. This finding illustrates that the trigger threshold alone does not give an adequate description of the performance of a radio neutrino detector. The bandwidth of the trigger circuit must be considered as well. We find that a reduced bandwidth of the trigger circuit strongly improves the sensitivity of the detector.

At first, this conclusion may seem counterintuitive because if a neutrino interaction is observed directly on the Cherenkov cone, the frequency content increases up to frequencies of $1 \mathrm{GHz}$. To first order, the signal amplitude increases linearly with frequency up to a cutoff frequency after which 


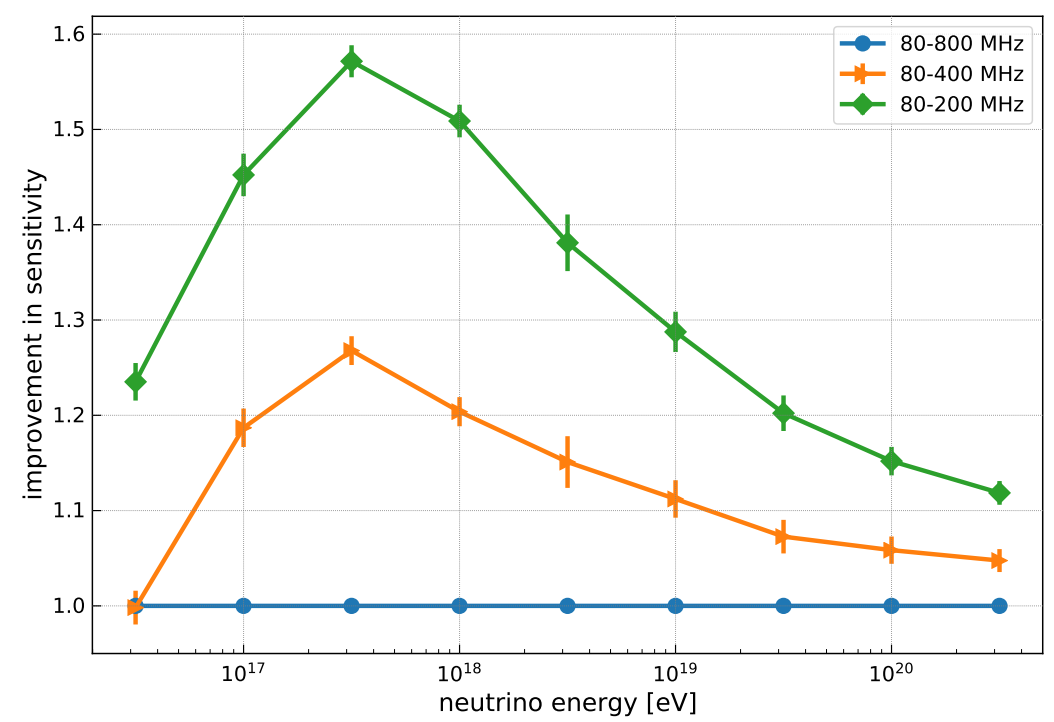

Figure 2: Impact of bandwidth on neutrino efficiency using the high/low trigger. The y-axis shows the improvement in sensitivity with respect to the largest studied bandwidth of $80 \mathrm{MHz}-800 \mathrm{MHz}$.

the amplitude decreases quickly. Thus, for neutrino interactions observed on the Cherenkov cone, a larger bandwidth detects more power which should result in better sensitivity.

However, there are several more relevant factors working against that. First, with increasing angular distance between the observation angle and the Cherenkov angle, the cutoff frequency drops quickly into the hundred $\mathrm{MHz}$ range (see e.g. discussion in [10, 12]). For a given amplitude at the detector, the geometry of off-cone events is more favorable and leads to a greater number of observed events.

Second, the effective area of radio antennas typically scale with the square of the wavelength of the incoming signal, leading to more detected power at lower frequencies. The relevant quantity is the vector effective length which is the proportionality factor between the incident electric field and the voltage output of the antenna. The vector effective length as function of frequency $\mathcal{H}(f)$ is related to the gain $G(f)$ as (see appendix of [11] for details)

$$
\mathcal{H}(f) \propto \frac{1}{f} \sqrt{G(f)} .
$$

Thus, higher frequencies are naturally suppressed via $1 / f$ and the antennas used in current Askaryan detectors like ARA and ARIANNA have the peak response between $100 \mathrm{MHz}$ and $200 \mathrm{MHz}$.

Third, the RMS noise is typically flat in frequency. Then, the RMS noise is proportionally to the square root of the bandwidth. Thus, reducing the bandwidth will substantially reduce the RMS noise. For example reducing the bandwidth from $720 \mathrm{MHz}(80 \mathrm{MHz}-800 \mathrm{MHz})$ to $120 \mathrm{MHz}$ $(80 \mathrm{MHz}-200 \mathrm{MHz})$ reduces the RMS noise by a factor 2.5 .

To visualize these effects, we calculated the average frequency spectrum of triggered events which is shown in Fig. 3. For all simulated neutrinos with an energy of $10^{17} \mathrm{eV}$ that pass the high/low trigger with a bandwidth of $80 \mathrm{MHz}$ to $800 \mathrm{MHz}$, we took the frequency spectrum after the antenna response was applied to the simulated Askaryan signals, normalized the power spectrum 


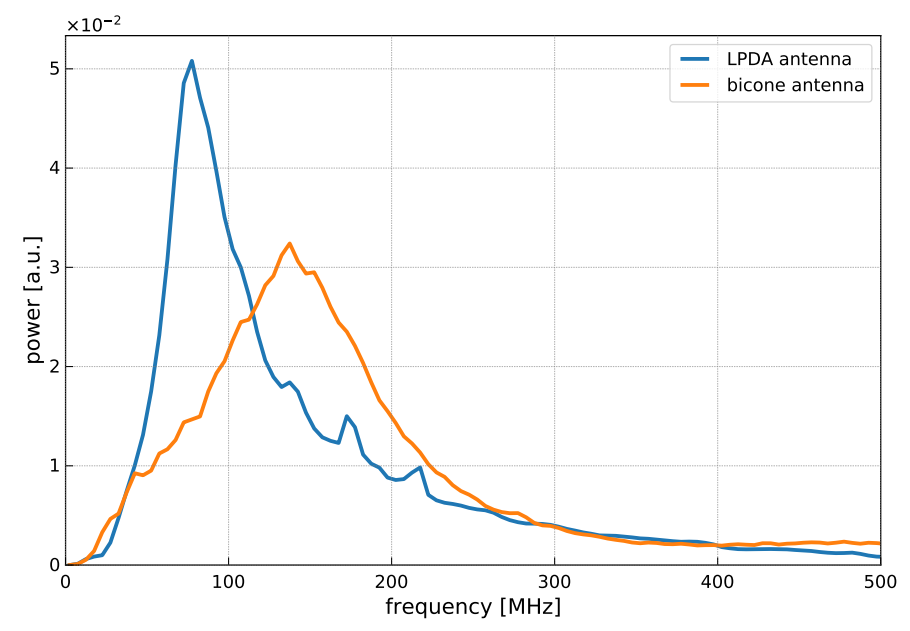

Figure 3: Averaged measured frequency spectrum of simulated neutrinos with an energy of $10^{17} \mathrm{eV}$. The blue curve shows the average frequency spectrum recorded with an LPDA antenna and the orange curve shows the average frequency spectrum recorded with a bicone antenna for the same events.

to the same integral, and summed up the contributions of all events. We observe that the resulting frequency spectrum is dominated by low frequencies. In case of an LDPA antenna, the spectrum is peaked at $80 \mathrm{MHz}$ which corresponds to the peak sensitivity of the LDPA antenna. We also analyzed the frequency spectrum measured by a bicone antenna where the peak in the frequency spectrum is at around $150 \mathrm{MHz}$. This is close to the peak sensitivity of $180 \mathrm{MHz}$ of the bicone antenna (cf. [11]). In both cases, most signal power is concentrated at low frequencies.

In summary, a narrow bandwidth trigger will reduce both the collected power of the Askaryan signal and the thermal noise. Due to the concentration of signal at the lower frequencies, especially for geometries off the Cherenkov cone, the reduction in noise power is greater than the reduction of signal power. Consequently, the sensitivity of the detector increases with decreasing bandwidth of the trigger. The effect is most pronounced at intermediate neutrino energies where many events are detected just above the detection threshold. At higher neutrino energies the signal amplitudes are often higher than the threshold which makes the high energies less dependent on changes of the trigger threshold. The weaker improvement at $10^{16.5} \mathrm{eV}$ is more difficult to explain. A likely cause is that at these low energies, only showers that are observed directly on the Cherenkov cone produce a large-enough signal where the benefit of a reduced bandwidth is smaller.

We studied a further fine tuning of the optimal bandwidth by e.g. decreasing the lower cutoff frequency to $50 \mathrm{MHz}$, and by further variations of the upper cutoff frequency (e.g. to $150 \mathrm{MHz}$ ) but did not find a significant additional gain in sensitivity. This is because an additional (small) variation in available bandwidth will not change the noise level significantly, compared to the factor of 2.5 reduction that is achieved by reducing the upper cutoff frequency from $800 \mathrm{MHz}$ to $200 \mathrm{MHz}$. In practice, also RFI noise sources at lower frequencies discourage lowering the low frequency cutoff further. 


\section{Conclusions}

Ultra-high energy (UHE) neutrinos can be measured cost-efficiently with an in-ice array of radio antennas that detect the Askaryan radiation following a neutrino interaction in the ice. The frequency content of these Askaryan signals ranges from around hundred $\mathrm{MHz}$ to a $\mathrm{GHz}$. The technology has been successfully explored in test-bed arrays, and large detector arrays are currently in the planning and construction phase. The low flux of UHE neutrinos makes it vital to optimize the sensitivity of each detector station as much as possible.

In this paper, we presented a simple way to increase the sensitivity by more than $50 \%$ by just optimizing the bandwidth of the trigger channels. We find that reducing the bandwidth from $80-800 \mathrm{MHz}$ that is currently being used to a much smaller bandwidth of $80-200 \mathrm{MHz}$ increases the sensitivity substantially. For the high/low trigger we find an increase of $50 \%$ between the relevant neutrino energies of $10^{17} \mathrm{eV}$ and $10^{18} \mathrm{eV}$.

The findings also apply to the interferometric phased array trigger concept which produces a synthesised waveform with increased signal-to-noise ratio by coherently adding the signals from multiple antennas. The same trigger optimizations discussed here can be applied to the synthesised waveform. A smaller bandwidth also comes with the additional practical benefit of smaller digitization rates. The phased array requires a real time digitization. Thus, reducing digitization speeds from several $\mathrm{GHz}$ to $400 \mathrm{MHz}$ (which corresponds to the Nquist frequency at $200 \mathrm{MHz}$ ) will save cost, power and data rates. These optimizations were already performed for the interferometric phased array trigger system of the Radio Neutrino Observatory in Greenland (RNO-G) [3].

In this work, only the first trigger stage was considered. To reduce the burden on input/output speeds, communication bandwidth, data storage and computing requirements, it is advisable to further reduce the trigger rate with secondary trigger stages that reject thermal noise while keeping Askaryan signals with high efficiency. Several approaches are currently being studied, among others neural networks that have the advantage of fast and constant processing speed and would allow to filter data in real-time [7].

\section{Acknowledgements}

The authors would like to thank their colleagues from the radio neutrino community for the lively discussions and input on this manuscript. We thank Allan Hallgren for providing detailed feedback on the manuscript. The author acknowledge funding from the German Research Foundation (DFG) under grant GL 914/1-1.

\section{References}

[1] ARIANNA collaboration, A search for cosmogenic neutrinos with the ARIANNA test bed using 4.5 years of data, Journal of Cosmology and Astroparticle Physics 03 (2020) 053 [1909.00840].

[2] ARA collaboration, Performance of two Askaryan Radio Array stations and first results in the search for ultrahigh energy neutrinos, Physical Review D 93 (2016) 082003 [1507.08991]. 
[3] RNO-G collaboration, Design and sensitivity of the radio neutrino observatory in Greenland (RNO-G), Journal of Instrumentation 16 (2021) P03025 [2010 . 12279].

[4] S. Barwick for the ARIANNA Collaboration, Science Case and Detector Concept for the ARIANNA High Energy Neutrino Telescope at Moore's Bay, Antarctica, Proc. 37th ICRC 2021, Berlin, Germany, PoS(ICRC2021)1037.

[5] S. Hallmann for the IceCube-Gen2 Collaboration, Sensitivity studies for the IceCube-Gen2 radio array, Proc. 37th ICRC 2021, Berlin, Germany, PoS(ICRC2021)468.

[6] ARA collaboration, Design and performance of an interferometric trigger array for radio detection of high-energy neutrinos, Nuclear Instruments and Methods in Physics Research A 930 (2019) 112 [1809.04573].

[7] A. Anker for the ARIANNA Collaboration, A novel trigger based on neural networks for radio neutrino detectors, Proc. 37th ICRC 2021, Berlin, Germany, PoS(ICRC2021)877 .

[8] C. Glaser and S. W. Barwick, An improved trigger for askaryan radio detectors, Journal of Instrumentation 16 (2021) T05001 [2011.12997].

[9] ARIANNA collaboration, Neutrino vertex reconstruction with in-ice radio detectors using surface reflections and implications for the neutrino energy resolution, Journal of Cosmology and Astroparticle Physics 11 (2019) 030 [1909.02677].

[10] C. Glaser et al., NuRadioMC: Simulating the radio emission of neutrinos from interaction to detector, European Physical Journal C80 (2020) 77 [1906. 01670].

[11] C. Glaser, A. Nelles, I. Plaisier, C. Welling, S. W. Barwick, D. García-Fernández et al., NuRadioReco: A reconstruction framework for radio neutrino detectors, The European Physical Journal C 79 (2019) [1903.07023].

[12] J. Alvarez-Muñiz, R. A. Vázquez and E. Zas, Calculation methods for radio pulses from high energy showers, Physical Review D 62 (2000) . 